\title{
Analytic Model for Conduction Current in AlGaN/GaN HFETs/HEMTs
}

\author{
Danqiong Hou, Griff L. Bilbro, and Robert J. Trew \\ Department of ECE, North Carolina State University, Raleigh, NC 27695-7911, USA \\ Correspondence should be addressed to Griff L. Bilbro, glb@ncsu.edu
}

Received 10 August 2011; Accepted 28 October 2011

Academic Editor: Narayanan Balasubramanian

Copyright $\odot 2012$ Danqiong Hou et al. This is an open access article distributed under the Creative Commons Attribution License, which permits unrestricted use, distribution, and reproduction in any medium, provided the original work is properly cited.

We have developed a new, zone-based compact physics-based AlGaN/GaN heterojunction field-effect transistor (HFET) model suitable for use in commercial harmonic-balance microwave circuit simulators. The new model is programmed in Verilog-A, an industry-standard compact modeling language. The new model permits the dc, small-signal, and large-signal RF performance for the transistor to be determined as a function of the device geometric structure and design features, material composition parameters, and dc and RF operating conditions. The new physics-based HFET model does not require extensive parameter extraction to determine model element values, as commonly employed for traditional equivalent-circuit-based transistor models. The new model has been calibrated and verified. We report very good agreement between simulated and measured dc and RF performance of an experimental C-band microwave power amplifier.

\section{Introduction}

AlGaN/GaN heterojunction field-effect transistors (HFETs) are promising RF transistors for use in high-power and high-frequency circuit applications. These HFETs possess a combination of high current density capability and high breakdown voltage due to the desirable physical properties of the materials, such as high critical electric field for breakdown, high electron mobility and saturated carrier velocity, high carrier density in the channel, lower dielectric constant compared to the conventional materials, and high thermal conductivity. These parameters permit the HFET to operate at high RF voltage and current, which results in high power operation at high frequency $[1,2]$. The technology for fabricating devices and circuits in $\mathrm{AlGaN} / \mathrm{GaN}$ is developing rapidly [3-6] and this rapid development is creating a need for improved device models. RF power amplifiers based on AlGaN/GaN HFETs are now commercially available from several companies, including Nitronex, RFMD, and TriQuint. However, to date, no commercially available HFET model for use in harmonic-balance circuit simulators exists that can predict the large-signal RF operation of an HFET or an MMIC before the active device is fabricated, characterized, and fitted.
The basic structure for an HFET is shown in Figure 1. The dc and RF performance of the transistor varies with the physical dimensions and material properties. However, the sensitivity of RF power performance to the physical parameters will vary, depending upon the particular parameter, and variations in some parameters (e.g., the gate length, $l_{g}$ ) have a more significant effect upon device performance than others. These specific parameter sensitivities are not easy to determine. Consequently, MMIC designers cannot consider the physical parameters of the device when designing circuits. They can only simulate circuits containing transistors with known and defined equivalent circuit compact models, which preclude the use of harmonic balance simulators for use in device optimization. In this report, we introduce an analytic HFET model that permits the codesign and optimization of active HFETs and passive elements in an MMIC environment, which will enhance and speed integrated circuit development.

Reported HFET dc models include empirical models $[7,8]$, two-dimensional multiphysics solvers $[9,10]$, and compact physics-based models [11-16]. Compact equivalent circuit models are suitable for simulating HFET circuits, but not before the HFET devices have been fabricated. Twodimensional solvers can predict the dc $I-V$ characteristics of 


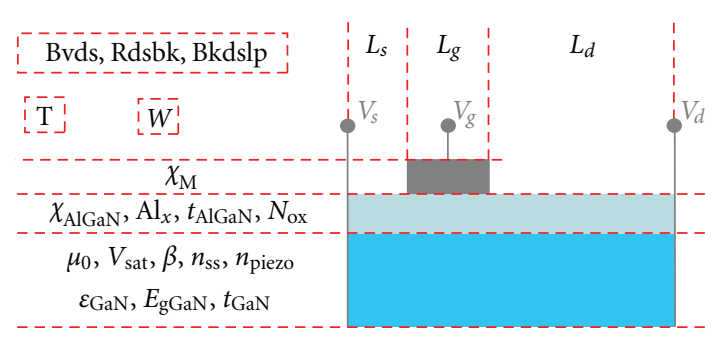

Figure 1: Schematic cross-section of a basic AlGaN/GaN HFET structure, showing the physical parameters of the NCSU HFET model. Four describe layout: $W, l_{s}, l_{g}, l_{d}$. Four describe the barrier layer, $\chi_{\mathrm{AlGaN}}, \mathrm{Al}_{x}, t_{\mathrm{AlGaN}}, n_{\mathrm{ox}}$. Seven describe the $\mathrm{GaN}$ buffer, $\varepsilon_{\mathrm{GaN}}, E_{\mathrm{gGaN}}, t_{\mathrm{GaN}}$ for the GaN itself, three for electron transport in the GaN $\mu_{0}, v_{\text {sat }}, \beta$, and two for its interface with AlGaN, $n_{\mathrm{ss}}, n_{\text {piezo }}$. The gate metal is characterized by its electron affinity, $\chi_{M}$.

an HFET and even its small-signal RF parameters, but they are difficult to employ in real time within a harmonic balance circuit simulator since run-time interpolation of a database of precomputed solutions is cumbersome [17, 18]. Compact physics-based models, however, can run in harmonic balance solvers because they are analytic and therefore sufficiently efficient in computation time requirements that they can predict the operation of an RF HFET under large-signal RF drive conditions. Previous work has demonstrated the facility and accuracy of this approach $[19,20]$. Unfortunately, however, this capability is not generally available, because the previously reported models could not be readily ported to commercial simulators. This report addresses that deficiency.

In this work, we report for the first time the implementation of a physics-based compact HFET model in Verilog$\mathrm{A}$, and we used Microwave Office (MWO) to compare its predictions against the RF power performance of an experimental HFET. MWO is an EDA package that is available from Applied Wave Research (AWR) Corporation. Our first-generation Verilog-A HFET model is a single, selfcontained Verilog-A module.

The new model is developed, based upon separating the conducting channel of the HFET into a series of zones, based upon operational physics [21]. The HFET model is generalized to suit different fabrication process by introducing a curvature parameter in the $v-E$ relationship [22] for electrons in the conducting channel of the HFET in order to control the sharpness of the knee of the $I-V$ characteristics. We also considered the electric field decay region in the drain access for triode operation to ensure continuity of the terminal characteristics. We modified the pinch off voltage to take into account different $\mathrm{Al}$ mole fractions in the AlGaN barrier layer in the AlGaN/GaN HFET. Finally, it is necessary to consider channel breakdown in order to accurately simulate Power Added Efficiency (PAE) at high RF power operation. We have written the model in Verilog-A language and implemented it in MWO. The model is verified by comparison of simulated and measured $\mathrm{dc}$ and RF large-signal performance for an $\mathrm{AlGaN} / \mathrm{GaN}$ HFET S-Band amplifier. Good agreement between the model simulation and the measurement data are obtained.

\section{Zone Model of the AlGaN/GaN HFET}

As previously indicated, the model is formulated based upon separating the conducting channel of the HFET into a series of five zones, which are defined based upon the physics that dominate within each zone. The model operates in two modes, triode and saturation. The transition between the two modes is smooth and dependent upon device design and operation criteria. Figure 2 shows the salient features of the zone model in its two operating modes. The typical electron is assumed to remain in the same longitudinal crosssection of the device on its path from source to drain. This defines the $x-y$ plane, where $x$ is measured from the source electrode and $y$ is measured down from the $\mathrm{AlGaN} / \mathrm{GaN}$ interface. For each operating mode, the path between source and drain is segmented into a few contiguous intervals. In each interval, the physical operation is determined by twodimensional numerical simulations and this information is used to develop an analytic physical model for that particular zone. The 2D simulations reveal the fundamental operation of each zone and permit the simplified analytic physical model to be derived. At the boundaries between adjacent zone intervals, the physical operation changes and the zones are interfaced by enforcing continuity of the electric potential values and derivatives.

Associated with each interval, we define its zone as the interval itself, initialization of the distance, potential, and electric field $\{x, V, E\}$. We also define a rule for terminating the interval, and a sequence of operations to compute $\{x, V, E\}$ at the end-point and within it, as appropriate.

When the HFET is in triode operation, it can be modeled with four zones. In saturated operation, the model requires five zones. For each operating mode, the terminal characteristics of the device must be consistent with a simultaneous solution of all the zones that exist in that mode.

Fortunately, it is possible to compute this simultaneous solution efficiently by setting up equations for the zones from left to right in sequence, as we will now show. Each zone is solved in three steps. First, the distance, potential, and electric field parameters $\{x, V, E\}$ are initialized at its left-hand boundary by applying its left-hand initialization rule to the final value of the triple from the preceding zone. Second, the nominal model in the zone is used to advance the parameters $\{x, V, E\}$ until the termination condition is detected. Third, the final values of $\{x, V, E\}$ are recorded and are available for the subsequent zone and elsewhere in that evaluation of the controlled voltage source.

In either of the two operating modes, the terminal characteristics can be qualitatively related to the physics of each zone. At zero drain-to-source bias $V_{\mathrm{ds}}=0$, the channel consists of three zones, the source and drain access regions and the region beneath the gate. We denote the source and drain access regions as the source neutral zone (SNZ, or Z1) and the drain neutral zone (DNZ, or Z5), respectively, to emphasize their net Coulombic neutrality which results from the approximate equality of the sheet charge density of the 2DEG in these two regions and the polarization charge density. We denote the region beneath the gate as the intrinsic FET zone (IFZ, or Z2). 


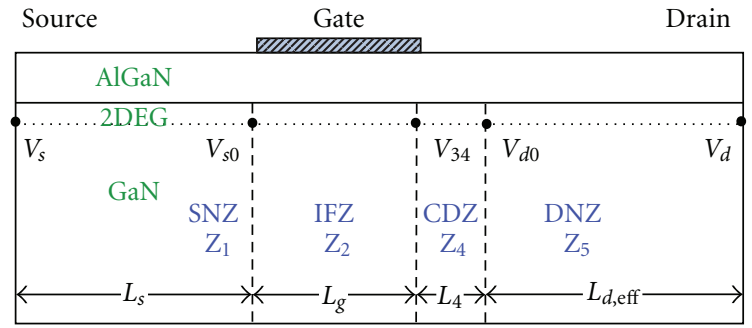

(a)

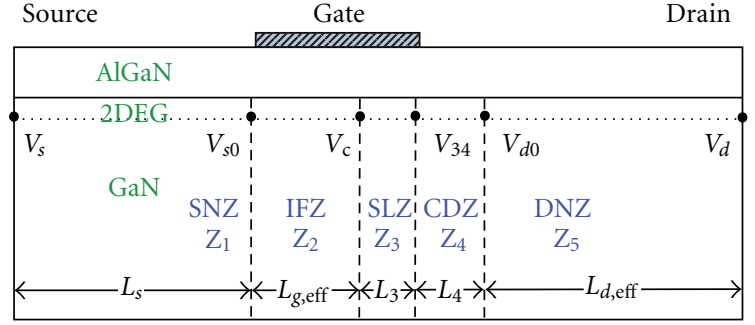

(b)

FIGURE 2: Cross-section of HFET model (a) triode operation with its four physical zones and the voltages at the boundaries between them. (b) Saturated operation with its 5 physical zones. Except for Z4, the dotted line indicates the electron path in the 2DEG just below the $\mathrm{AlGaN} / \mathrm{GaN}$ interface. In $\mathrm{Z} 4$, the 2DEG is disrupted and the electrons disperse away from the interface to form a net space charge in the $\mathrm{GaN}$, as discussed in the text.

For positive $V_{\mathrm{ds}}$, a charge-deficit zone (CDZ, or $\left.\mathrm{Z} 4\right)$ forms in the drain access region close to the gate edge. The positive net charge in this partially depleted region (Z4) smoothly reduces the magnitude of the electric field, in accordance with Poisson's equation. In the triode mode, the length of the Z4 is short and the voltage drop across it is typically less than a volt, but it is required for continuity of $V_{\mathrm{ds}}$ at the transition to saturated operation at $V_{\mathrm{ds}}=V_{\mathrm{dsat}}$. At fixed gate bias, the length of the CDZ zone increases with drain bias, which reduces the length of the DNZ zone, since the sum of their lengths equals the gate-to-drain spacing, $L_{d}$.

For saturated operation, $V_{\mathrm{ds}}>V_{\mathrm{dsat}}$. Electrically in the $V_{\mathrm{ds}}-I_{\mathrm{ds}}$ plane, the transition from triode to saturation operation occurs at the knee of the $I-V$ curve, where the slope of the curve flattens. Physically, saturated operation begins when the magnitude of the longitudinal electric field in the channel at the drain-side gate edge (denoted as $E_{34}$ ) first exceeds the critical field, $E_{c}$, which effectively pinches the 2DEG off by flat banding the quantum well.

With increasing $V_{\mathrm{ds}}$, the electric field $\left(E_{34}>E_{c}\right)$ at the drain-side edge of the gate continues to increase and the location where $E$ first exceeds $E_{c}$ moves toward the source. Under the gate, we denote the location where $E=E_{c}$ as $x_{c}$ and the voltage there as $V_{c}$. In the region $x_{c}<x<l_{s}+l_{g}$, electrons are repelled away from the $\mathrm{AlGaN} / \mathrm{GaN}$ interface to form the velocity-saturated space-charge-limited zone (SLZ, or Z3). This SLZ extends longitudinally to the gate edge and typically extends down to the substrate because the gate repels electrons when $V_{g}<V$, so that the gradual channel Approximation (GCA) fails and the magnitude of the field increases rapidly with distance, $x$.

With increasing $V_{\mathrm{ds}}$, the length of the SLZ zone increases, which reduces the length of the IFZ zone under the gate since the sum of their lengths is constrained to be $l_{g}$. In the drain access region, the length of the CDZ zone increases simultaneously with increasing $V_{\mathrm{ds}}$, which reduces the length of the DNZ zone, since the sum of their lengths is constrained to equal the gate-to-drain distance.

The gradual channel approximation (GCA) is readily adapted to zones Z1, Z2, and Z5. In zone Z3, the GCA fails because the carrier trajectories are not one dimensional as discussed above. In zone Z4, the 2DEG has reestablished itself, but the sheet charge density of the 2DEG is insufficient to neutralize the fixed polarization sheet charge and electron velocity in this $2 \mathrm{DEG}$ is effectively saturated.

To the best of our knowledge, this charge deficit zone (or zone Z4) is unique to AlGaN/GaN HFETs, but it can dominate device operation over part of the RF cycle. In zone $\mathrm{Z} 4$, the 2DEG is stable but is incompletely filled. The length of zone Z4 is approximately proportional to the difference $E_{34}-E_{c}$, but $E_{34}$ increases with $I_{\mathrm{ds}}$ until $E_{34} \geq E_{\mathrm{BD}}$ when impact ionization in the channel produces unacceptable breakdown effects at the device terminals. In $\mathrm{GaN}$, the breakdown electric field $E_{\mathrm{BD}} \gg E_{c}$ is about two orders of magnitude larger than the electric field $E_{c}$ at which velocity saturation begins. Therefore, the maximum length of this region is proportional to $E_{\mathrm{BD}}-E_{c}$ which is exceptionally large for $\mathrm{GaN}$. Electrons enter zone $\mathrm{Z} 4$ with their velocity saturated and the transistor effect of the gate region limits the current almost independently of the local electric field, even when it is two orders of magnitude larger than $E_{c}$, as it can be in GaN before its breakdown occurs at very high $V_{\mathrm{ds}}$. This combined effect keeps zone Z4 partially depleted, so that at fixed gate bias, the voltage dropped across zone Z4 increases approximately quadratically with its length, which adjusts itself to gradually reduce $E$ from $E_{34}$ to $E_{s}$, where the field-dependent mobility of the electrons is sufficiently high to reestablish net Coulombic neutrality at the given $I_{\mathrm{ds}}$. Over the majority of a typical RF cycle, most of $V_{\mathrm{ds}}$ drops across the CDZ zone.

\section{Generalized Velocity-Field Relationship for Carrier Electrons}

Theoretical investigations of electron dynamics using Monte Carlo techniques have determined velocity-field characteristics associated with GaN materials. These theoretical simulations show that the electron drift velocity initially increases with the applied electric field but reaches a peak value, after which it gradually decreases to a saturated value at a high electric field [23]. The peak value decreases with increasing doping concentration and temperature [24]. Some $v$-E relationships for electron transit in a 2DEG for the $\mathrm{AlGaN} / \mathrm{GaN}$ structure have been reported [23, 25]. However, no evidence of a velocity overshoot is apparent in the terminal characteristics of devices that we have considered. In fact, an equilibrium $v$ - $E$ characteristic has proved adequate 


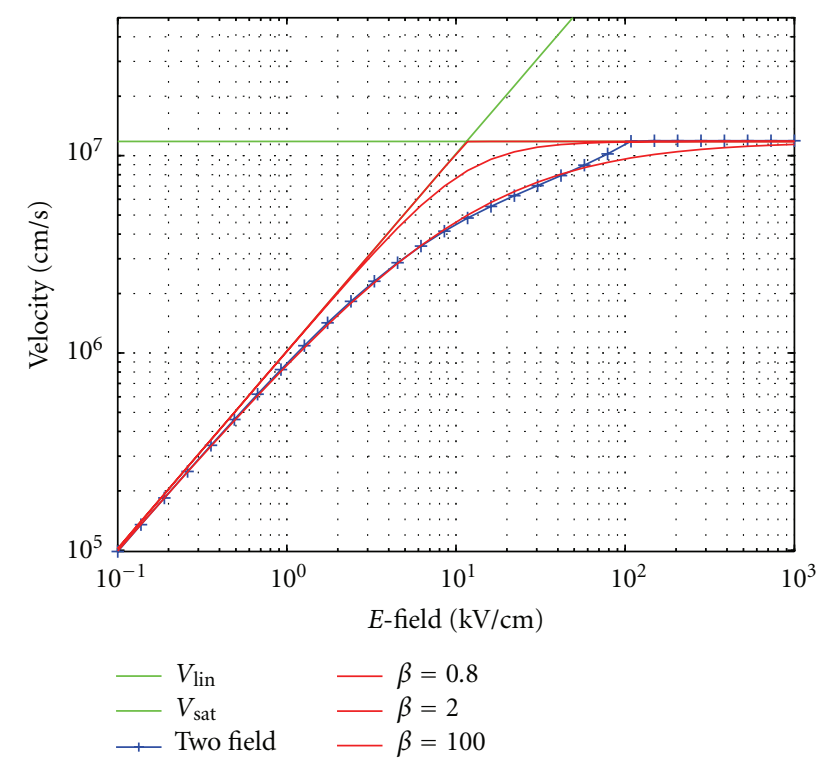

FIGURE 3: Various $v$-E models: two-piece velocity model (green), two-field model (blue) and $v(E)$ as in (1) for $\beta=0.8,2,100$ (red). $\beta=0.8$ fits the two-field model.

to accurately simulate experimental results and we find that (1) accurately simulates the dc and RF currents that flow in experimental AlGaN/GaN HFETs.

The curvature of the transition from the low-field region to the high-field region is important in calculating the knee region of the dc current-voltage relationship for the device. We have found that the same $v-E$ model (1) accurately simulates both the dc and large-signal RF operation. Consequently, we have chosen (1) for the velocity-field relationship because its transition is smooth but adjustable. Figure 3 also compares the $v-E$ relationship in (1) to the two-field relationship.

The $v$-E relationship we adopt is shown as

$$
v(E)=\frac{\mu_{0} E}{\left(1+\left(E / E_{c}\right)^{\beta}\right)^{1 / \beta}},
$$

where we define

$$
E=-E(x)=\frac{d V}{d x}
$$

as the negative of the usual longitudinal electric field and we regard it as a function of distance from the source electrode. In (2), $\mu_{0}$ is the low-field electron mobility:

$$
E_{c}=\frac{v_{\mathrm{sat}}}{\mu_{0}}
$$

is the critical electric field marking the onset of the high-field region:

$$
v_{\mathrm{sat}}=v(E \longrightarrow \infty)
$$

is the asymptotic saturated velocity, and $\beta$ controls the curvature of the knee of the $v$-E curve. For the experimental data that we have analyzed, this curvature parameter has varied over the interval $1 \leq \beta \leq 2$, so that the critical electron velocity $v\left(E_{c}\right)=2^{-1 / \beta} v_{\text {sat }}$ depends on $\beta$ as well as $v_{\text {sat }}$. In our investigations, the values of the $\mu_{0}, v_{\text {sat }}, \beta$ parameters can be determined by adjusting the estimated physical-based values so that the simulated terminal $I-V$ characteristics match the measured $I-V$ data for an HFET.

Figure 3 shows how $\beta$ controls the curvature of the knee of (1) without changing the low-field and high-field regions of the $v-E$ model. In the limit of large $\beta$, this $v-E$ curve approaches a piecewise linear $\nu$ - $E$ model. For the choice $\beta=$ 0.8 , this $v$-E curve approaches the two-field model with the same parameters as in [15].

We estimate the saturated velocity $v_{\text {sat }}$, the low-field mobility $\mu_{0}$, and the rate of transition from $v \approx \mu_{0} E$ to $v \approx v_{\text {sat }}$ from simultaneous fits of dc and RF measurements when we use the velocity-field relationship in (1). The initial value for the electron mobility at low electric field is generally determined from measured Hall mobility data. This value may be slightly varied in order to accurately simulate the measured dc $I-V$ data in the linear region in some cases but does not significantly vary from the Hall values, and often the measured Hall value is found to produce excellent results.

\section{Pinch-Off Voltage}

The conducting channel in an AlGaN/GaN HFET is formed from the 2DEG just below the interface of the AlGaN barrier layer that is grown on a GaN layer. This 2DEG conducting channel is formed by spontaneous and piezoelectric polarization effects at the $\mathrm{AlGaN} / \mathrm{GaN}$ interface [26]. Figure 2 shows the cross-sectional view of an HFET. The sheet charge density of this 2DEG channel is determined by the aluminum percentage and the thickness of the AlGaN layer. The sheet charge density can be modulated by the deposition of a gate electrode on the AlGaN surface and by the electric voltage $V_{g}$ that is applied to the gate electrode. When $V_{g}$ is sufficiently negative, the channel vanishes everywhere under the gate and, in particular, at the source-side edge of the gate, which determines the pinch-off voltage $V_{\text {th }}$ of the AlGaN/GaN HFET. The pinch-off voltage is conventionally written

$$
V_{\mathrm{th}}(m)=\Phi_{\mathrm{th}}(m)-\Delta E_{c}(m)-\frac{q N_{D} d_{d}^{2}}{2 \varepsilon(m)}-\frac{\sigma(m)}{\mathcal{E}(m)} d_{\mathrm{eff}}
$$

as a function of mole fraction $m$ of aluminum in the $\mathrm{Al}_{m} \mathrm{Ga}_{1-m} \mathrm{~N}$, but $V_{\text {th }}$ also depends on the effective thickness $d_{\text {eff }}$ of the AlGaN barrier, its Schottky barrier height $\Phi_{\text {th }}(m)$, its electric permittivity $\varepsilon(m)$, and its doping $N_{D}$, as well as the conduction band offset $\Delta E_{c}(m)$ at the AlGaN/GaN interface and the combined piezoelectric/spontaneous polarization sheet charge $\sigma(m)$. In (5), the dielectric constant and bandgap of $\mathrm{AlGaN}$ are expressed as [14]

$$
\begin{gathered}
\varepsilon\left(\mathrm{Al}_{m} \mathrm{Ga}_{1-m} \mathrm{~N}\right)=\varepsilon(\mathrm{GaN})-1.2 m, \\
E_{g}\left(\mathrm{Al}_{m} \mathrm{Ga}_{1-m} \mathrm{~N}\right)=E_{g}(\mathrm{GaN})+2.32 m+0.0796 m(1-m),
\end{gathered}
$$

and the Schottky barrier is expressed as

$$
\Phi=0.91+2.44 m
$$


and the band offset is given by the expression:

$$
\Delta E_{c}=0.7\left(E_{g}\left(\mathrm{Al}_{m} \mathrm{Ga}_{1-m} \mathrm{~N}\right)-E_{g}(\mathrm{GaN})\right) .
$$

\section{Physics of the Zones}

5.1. Zone Z1, the Source Neutral Zone, or SNZ. In zone Z1 (and also in zone Z5), the current at location $x$ is conducted by the 2DEG, but for steady-state operation, $I(x)=I_{\mathrm{ds}}$ cannot depend on $x$, so that

$$
I_{\mathrm{ds}}=W q n_{s}(x) v(E(x)),
$$

where $I_{\mathrm{ds}}$ is the drain current, $W$ is the gate width, $q$ is the fundamental charge, and $n_{s}(x)$ is the local electron density of the 2DEG. In the source access region, $n_{s}(x)=n_{\mathrm{ss}}$ is fixed at $n_{\mathrm{ss}}=\sigma(m) / q$ to neutralize $\sigma(m)$, so that (9) and (1) imply that

$$
E(x)=\frac{E_{c} I_{\mathrm{ds}}}{\left(I_{\max }^{\beta}-I_{\mathrm{ds}}^{\beta}\right)^{1 / \beta}} \equiv E_{s}
$$

is constant with respect to $x$, where $E_{s}$ is the value of that constant at a given $I_{\mathrm{ds}}$ and $I_{\max }=W q n_{\mathrm{ss}} v_{\mathrm{sat}}$ is a convenient scale factor for $I_{\mathrm{ds}}$ for a given HFET.

The electric field $E_{s}$ typically occurs near the drain electrode in zone Z5, the other neutral zone of the HFET. In contrast to the fixed length of zone $Z 1$, the length of zone Z5 is state dependent. Zone Z5 begins when the lateral field in zone Z4 has finally diminished to $E(x)=E_{s}$, which terminates zone $\mathrm{Z} 4$. Within both the source and drain neutral zones, electron transport is identical and $E_{s}$ sets the drift velocity $v\left(E_{s}\right)$ in both zones Z1 and Z5.

5.2. Zone Z2, the Intrinsic FET Zone, or IFZ. At the sourceside gate edge, electrons leave zone $\mathrm{Z1}$ and enter zone $\mathrm{Z} 2$. In zone $\mathrm{Z1}$, the voltage on the upper AlGaN surface follows the voltage $V(x)$ of the $2 \mathrm{DEG}$ at the $\mathrm{AlGaN} / \mathrm{GaN}$ interface. In zone $\mathrm{Z} 2$, however, the gate electrode holds the surface voltage at $V_{g}$.

In accordance with the GCA, we treat this gate region as an MIS capacitor and write the electron sheet density:

$$
n_{s}(x)=\frac{C_{\text {eff }}}{q}\left(V_{\mathrm{gt}}-V(x)\right)
$$

in terms of the effective gate voltage $V_{\mathrm{gt}}=V_{g}-V_{\mathrm{th}}(m)$, the effective gate-channel capacitance $C_{\text {eff }}=\varepsilon(m) / d_{\text {eff }}$, and the effective thickness $d_{\text {eff }}$ of the $\mathrm{Al}_{m} \mathrm{Ga}_{1-m} \mathrm{~N}$ barrier layer.

Electrons in zone Z2 drift toward the drain because $V(x)$ increases with $x$ in the channel, but this drift cannot persist past where $V(x)$ has risen enough to exceed $V_{\text {gt }}$. In triode operation, the channel fills the entire gate region. Following the GCA, we substitute (11) into (9) and use (2) to change the variable of integration from $x$ to $V$, so that in triode operation we find

$$
l_{g} I_{\mathrm{ds}}=\int_{V_{s 0}}^{V_{34}}\left(\left(W u_{0} C\left(V_{\mathrm{gt}}-V\right)\right)^{\beta}-\left(\frac{I_{\mathrm{ds}}}{E_{c}}\right)^{\beta}\right)^{1 / \beta} d V,
$$

where $l_{g}$ is the physical gate length, $V_{s 0}=E_{c} l_{s} I_{\mathrm{ds}} /$ $\left(I_{\max }^{\beta}-I_{\mathrm{ds}}^{\beta}\right)^{1 / \beta}$ is the voltage at the source-side gate edge, $V_{34}=V_{d}-\left(E_{c}\left(l_{d}-l_{4}\right) I_{\mathrm{ds}} /\left(I_{\max }^{\beta}-I_{\mathrm{ds}}^{\beta}\right)^{1 / \beta}\right)-(1 / 2) l_{4}^{2} k_{4}$ is the voltage at the drain-side edge of the gate in triode operation, and $l_{g}$ and $l_{4}$ are the lengths of drain access and zone Z4.

If $x=l_{s}+l_{g}$ while $E_{x}<E_{c}$, then the device is in triode mode; if $E_{x}=E_{c}$ before $x=l_{s}+l_{g}$, then the device is in saturated mode. In triode operation, zone $\mathrm{Z} 3$ does not affect the parameters $\{x, V, E\}$ because its thickness $l_{s}+l_{g}-x_{c}=$ 0 vanishes, zero voltage is dropped across the zone, and the electric field does not change. In saturation, zone $\mathrm{Z} 3$ is significant because $l_{s}+l_{g}-x_{c}>0$ when $I_{\mathrm{ds}}>I_{\text {sat }}$.

In saturated operation, $V_{34}$ and $E_{34}$ are still defined at the drain-side gate edge, but (12) is not valid there because the longitudinal electric field exceeds $E_{c}$ before exiting the gate region so that $E_{34}>E_{c}$ and $V_{34}>V_{c}$. In this case, the length of zone $\mathrm{Z} 2$ is less than $l_{g}$, and we write

$$
l_{g, \mathrm{eff}} I_{\mathrm{ds}}=\int_{V_{s 0}}^{V_{c}}\left(\left(W u_{0} C\left(V_{\mathrm{gt}}-V\right)\right)^{\beta}-\left(\frac{I_{\mathrm{ds}}}{E_{c}}\right)^{\beta}\right)^{1 / \beta} d V,
$$

where

$$
V_{c}=V_{\mathrm{gt}}-\frac{I_{\mathrm{ds}}}{W C v_{\mathrm{sat}}} .
$$

The transition of terminal characteristics from triode mode to saturated mode occurs when the drain current is high enough to make $V_{34}=V_{c}$ and $E_{34}=E_{c}$ at the gate edge. This $I_{\text {sat }}$ is determined by using (14) for the upper limit of (12) and solving

$$
L_{g} I_{\mathrm{sat}}=\int_{V_{s 0}}^{V_{c}}\left(\left(W u_{0} C\left(V_{\mathrm{gt}}-V\right)\right)^{\beta}-\left(\frac{I_{\mathrm{sat}}}{E_{c}}\right)^{\beta}\right)^{1 / \beta} d V,
$$

for $I_{\text {sat }}$.

5.3. Zone Z3, the Space-Charge-Limited Zone, or SLZ. The SLZ zone only occurs when the device enters saturation. We approximate the one-dimensional Poisson equation:

$$
\frac{\partial E}{\partial x}=\frac{q n_{s}(x)}{\varepsilon t(x)}=\frac{I_{\mathrm{ds}}}{\varepsilon W v(x) t(x)}=k_{3}(x)
$$

where $t(x)$ and $v(x)$ both vary with $x$. Comparison with two-dimensional finite-element simulations suggests that the product $t(x) v(x)$ is nearly constant:

$$
\frac{I_{\mathrm{ds}}}{I_{\max }}=\frac{n_{s}(x) v(x)}{n_{\mathrm{ss}} v_{\mathrm{sat}}},
$$

so that we can define the average electric field derivative:

$$
\overline{k_{3}}=\left\langle\frac{\partial E}{\partial x}\right\rangle=\left\langle\frac{I_{\mathrm{max}}}{\varepsilon W v(x) t_{\mathrm{GaN}}}\right\rangle \approx \frac{I_{\mathrm{max}}}{\varepsilon W v_{\mathrm{sat}} t_{\mathrm{GaN}}}=\frac{q n_{\mathrm{ss}}}{\varepsilon t_{\mathrm{GaN}}} .
$$

Integrating Poisson's equation once yields

$$
E_{c}+\int_{l_{g}-l_{3}}^{l_{g}} \frac{\partial E}{\partial x} d x+\int_{l_{g}}^{l_{g}+l_{4}} \frac{\partial E}{\partial x} d x=E_{s}
$$


and a second integration yields

$$
V_{c}+\iint_{l_{g}-l_{3}}^{l_{g}} \frac{\partial E}{\partial x} d x d x+\iint_{l_{g}}^{l_{g}+l_{4}} \frac{\partial E}{\partial x} d x d x+E_{s}\left(l_{d}-l_{4}\right)=V_{d}
$$

we can include zone $\mathrm{Z} 4$ in the sum $\sum_{j=1}^{j=5}(\Delta V)_{Z j}$ over all five zones of voltage increments for each zone to get $V_{d}\left(V_{\mathrm{gt}}, I_{\mathrm{ds}}\right)=\sum_{j=1}^{j=5}(\Delta V)_{Z j}$.

In the triode mode, we can define $(\Delta V)_{Z 3}=0$ in the same sum. To reexpress this result as the controlled current source $I_{\mathrm{ds}}\left(V_{\mathrm{gt}}, V_{\mathrm{ds}}\right)$ for given $V_{\mathrm{gt}}$ and $V_{\mathrm{ds}}$, we invert the sum $V_{\mathrm{ds}}\left(V_{\mathrm{gt}}, I_{\mathrm{ds}}\right)$ iteratively to find the $I_{\mathrm{ds}}$ corresponding to a given $V_{\mathrm{ds}}$.

5.4. Zone Z4, the Charge Deficit Zone, or CDZ. Zone Z4 occurs in both triode and saturated modes when $E_{34}>E_{c}$ as defined by (3). Since the surface voltage in zone Z4 is not clamped by the gate electrode, it rises with the channel potential $V(x)$ and the one-dimensional Poisson equation,

$$
\frac{\partial E}{\partial x}=\frac{q n_{s s}}{\varepsilon t(x)}-\frac{I_{\mathrm{ds}}}{\varepsilon W v(x) t(x)}=k_{4},
$$

can be used to obtain

$$
l_{4}=\frac{E_{34}-E_{s}}{k_{4}}
$$

assuming that $t(x)=t_{2 \mathrm{DEG}}$ and $v(x)=v\left(E_{c}\right)$. The voltage and electric field continuity conditions lead to the following two equations:

$$
\begin{gathered}
V_{34}+\iint_{l_{g}}^{l_{g}+l_{4}} \frac{\partial E}{\partial x} d x d x+E_{s}\left(l_{d}-l_{4}\right)=V_{d}, \\
E_{34}+\int_{l_{g}}^{l_{g}+l_{4}} \frac{\partial E}{\partial x} d x=E_{s} .
\end{gathered}
$$

5.5. Zone Z5, the Drain Neutral Zone, or DNZ. Within zone $\mathrm{Z5}$, the nominal electron physics are identical to that in zone $\mathrm{Z} 1$ but the zone length varies dynamically and satisfies different boundary conditions. As in zone Z1, overall charge neutrality prevails in zone Z5 and the current is described by (9) and (10), so the lateral electric field in zone Z5 is constant and coincides with Es in zone Z1. In the preceding zone $\mathrm{Z} 4, E_{x}>E_{s}$ but the electric field continuously varies from magnitude $E_{34}$ to $E_{s}$. In zone Z5, the electron transport model is similar as in zone Z1, the SNZ, but the length of zone $\mathrm{Z} 5$ varies dynamically. The lateral electric field in zone $Z 4$ varies and zone $Z 5$ begins when the lateral electric field in zone $\mathrm{Z} 4$ is reduced to its value in zone $\mathrm{Z} 1$.

It may be possible to fabricate an HFET with sufficiently short drain access region and to bias it at high enough drain voltage to deplete the entire drain access region, but we have not observed this in any device we have considered. Currently, we treat this possibility as an error condition.

\begin{tabular}{|c|c|c|}
\hline Parameter & Description & Value \\
\hline$\mu_{0}$ & Electron mobility & $1120 \mathrm{~cm}^{2} / \mathrm{V}-\mathrm{s}$ \\
\hline$v_{\text {sat }}$ & Saturation velocity & $1.195 \times 10^{-7} \mathrm{~cm} / \mathrm{s}$ \\
\hline$\beta$ & $\begin{array}{l}\text { Curvature parameter in } v \text { - } E \\
\text { relation }\end{array}$ & 1.45 \\
\hline$\varepsilon(\mathrm{GaN})$ & GaN permittivity & 10.1 \\
\hline$E_{g}(\mathrm{GaN})$ & Energy gap of GaN & $3.52 \mathrm{eV}$ \\
\hline$\chi_{\mathrm{AlGaN}}$ & Affinity of AlGaN & $3.8 \mathrm{eV}$ \\
\hline$\chi_{m}$ & Affinity of metal & $4.3 \mathrm{eV}$ \\
\hline $\mathrm{Al}_{x}$ & $\mathrm{Al}$ mole fraction in $\mathrm{AlGaN}$ & 0.3 \\
\hline$l_{g}$ & Gate length & $0.8 \mu \mathrm{m}$ \\
\hline$l_{s}$ & Length of the source access & $1.2 \mu \mathrm{m}$ \\
\hline$l_{d}$ & Length of the drain access & $2.0 \mu \mathrm{m}$ \\
\hline$W$ & Gate width & $400 \mu \mathrm{m}$ \\
\hline$t_{\mathrm{AlGaN}}$ & $\begin{array}{l}\text { Thickness of the AlGaN barrier } \\
\text { layer }\end{array}$ & $30 \mathrm{~nm}$ \\
\hline$t_{\mathrm{GaN}}$ & $\begin{array}{l}\text { Thickness of the GaN buffer } \\
\text { layer }\end{array}$ & $0.3 \mu \mathrm{m}$ \\
\hline$n_{\mathrm{ox}}$ & $\begin{array}{l}\text { Unintentional doping in } \mathrm{AlGaN} \\
\text { layer }\end{array}$ & $10^{16} \mathrm{~cm}^{-3}$ \\
\hline$n_{\mathrm{ss}}$ & $\begin{array}{l}\text { Polarization sheet charge } \\
\text { density }\end{array}$ & $7.1 \times 10^{12} \mathrm{~cm}^{-2}$ \\
\hline Piezo & Piezo charge density & $13 \times 10^{11} \mathrm{~cm}^{-2}$ \\
\hline$B V_{\mathrm{ds}}$ & Channel breakdown voltage & 39 \\
\hline$R_{\mathrm{dsbk}}$ & $\begin{array}{l}\text { Drain-to-source breakdown } \\
\text { resistance }\end{array}$ & 13 \\
\hline Bkdslp & Slope of $B V_{\mathrm{ds}}$ & 0 \\
\hline
\end{tabular}

The physics-based compact HFET model described above has been written in the Verilog-A language and implemented in MWO. The flowchart in Figure 4 shows the
TABLE 1: Input parameters for the AlGaN/GaN HFET model.

process to calculate the drain current. The model was verified by comparing the simulated and measured dc and largesignal RF performance for an HFET S-band amplifier. The parameters used in the model are listed in Table 1.

Note that the model requires twenty (20) input parameters, as compared to the eighty (80) parameters required to fit conventional equivalent-circuit-based models. The input parameters consist of physical dimensions, doping and thickness levels, and so forth, rather than equivalent circuit element values determined from parameter extraction procedures. The new model is, therefore, easier to define than traditional equivalent circuit transistor models.

\section{Simulation in Microwave Office}

To calibrate the model, we have compared the simulation with the measurement data for AlGaN/GaN HFETs of $0.8 \mu \mathrm{m}$ gate length and $400 \mu \mathrm{m}$ gate width. The curvature parameter $\beta$ in the $v$ - $E$ relationship is adjusted to be 1.45 for this device. The polarization sheet charge density is $7.1 \mathrm{e} 12 \mathrm{~cm}^{-2}$.

6.1. DC I-V Curves Simulated by MWO. The model is implemented in the commercial circuit simulator Microwave Office developed by AWR Corporation. The results are compared with the experimental measurements of an 


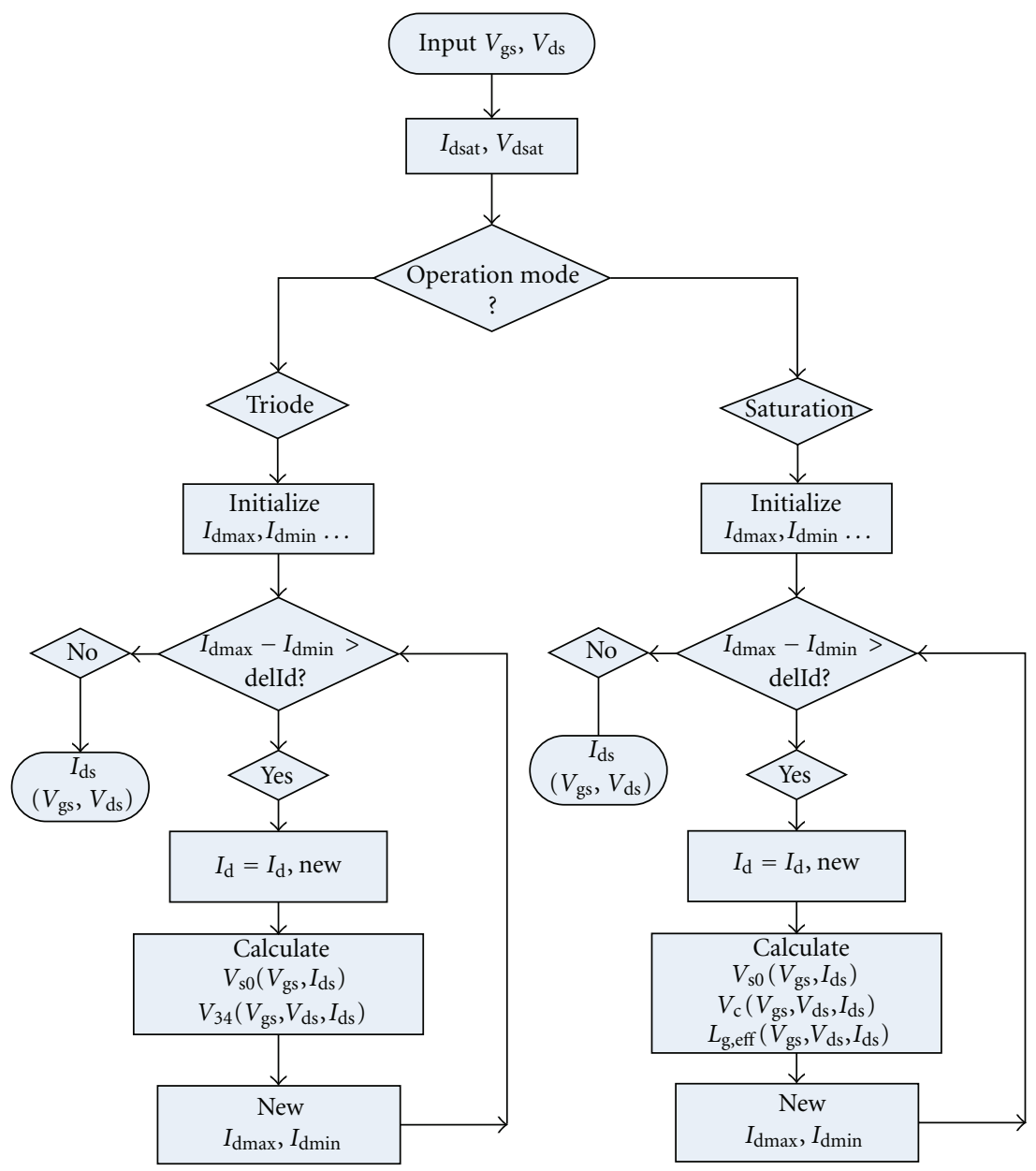

Figure 4: Flowchart to calculate the current as a function of $V_{\mathrm{gs}}$ and $V_{\mathrm{ds}}$.

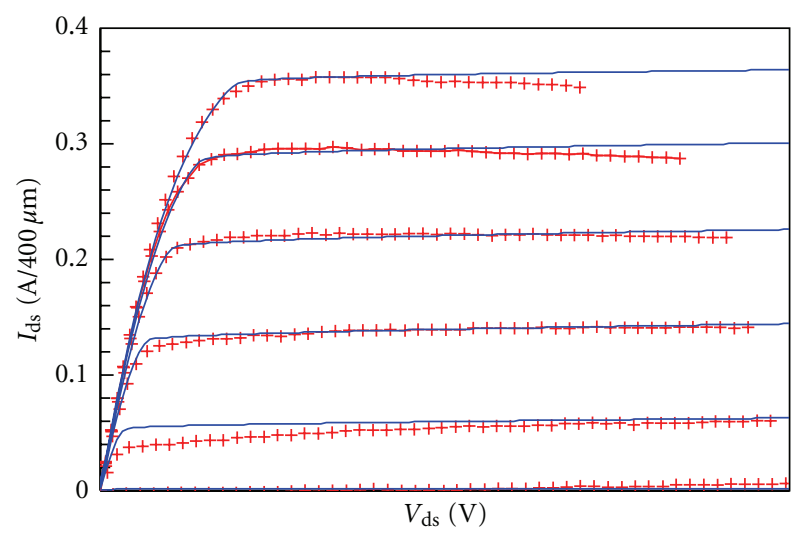

FIGURE 5: Current-voltage characteristics simulated by MWO for the HFET model (blue lines), along with experimental measurements (red lines) for an HFET with $0.8 \mu \mathrm{m}$ gate length and $400 \mu \mathrm{m}$ width. Each of the six curves corresponds to an integer $V_{\mathrm{gs}}$ value from $-4 \mathrm{~V}$ to $1 \mathrm{~V}$. All curves sweep $V_{\mathrm{ds}}$ from $0 \mathrm{~V}$ to $20 \mathrm{~V}$.

$l_{g}=0.8 \mu \mathrm{m}$ HFET. Figure 5 shows excellent agreement between the simulated results and measurement data.
The source and drain access regions, zones $\mathrm{Z1}$ and $\mathrm{Z} 5$, introduce an extrinsic resistance to the intrinsic HFET and will affect the performance of the HFETs. It has been previously shown that under high-current operation and large-signal RF drive, these resistances become nonlinear as space-charge-limited (SCL) current transport conditions are approached [20]. Both the source and drain resistances become nonlinear and increase with channel current. The effect is most apparent in the source region since the voltage drop across the source access region subtracts directly from the applied $V_{g}$ and limits the drain current of the HFET considerably before $I_{\mathrm{ds}}$ increases to $I_{\text {sat }}$. The drop across the drain access region lowers the slope of $I_{\mathrm{ds}}$ in the triode region and increases the drain bias required to achieve $I_{\text {sat }}$. In power microwave applications, using a constant resistance for either will typically overestimate the drain current at the upper left limit of the dynamic load line and with it, the output power. From (10) and the length $l_{s}$ of the source access region, we find

$$
R_{s}=\frac{V_{\mathrm{SNZ}}}{I_{\mathrm{ds}}}=\frac{E_{c} l_{s}}{\left(I_{\max }^{\beta}-I_{\mathrm{ds}}^{\beta}\right)^{1 / \beta}},
$$




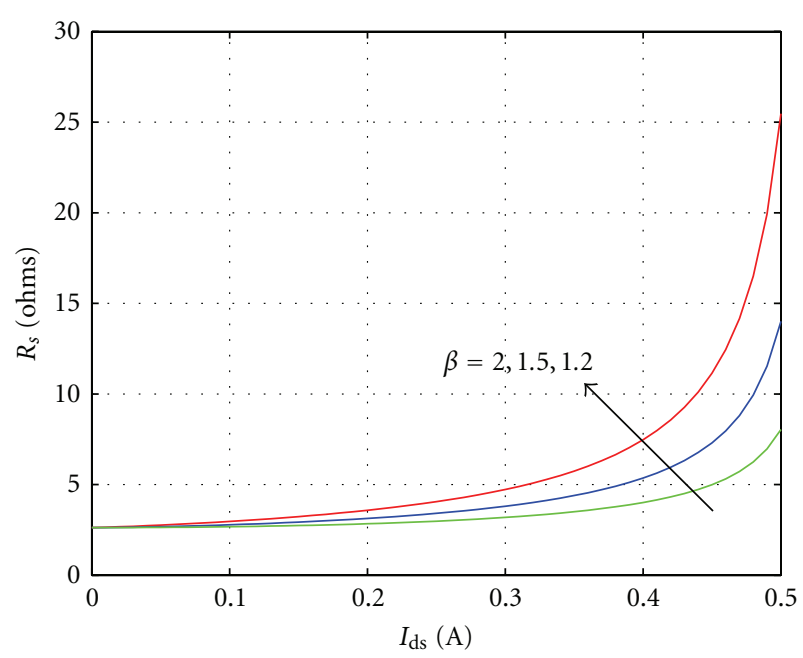

Figure 6: $R_{s}$ as a function of the conductance current with different beta. The values of beta are 2, 1.5, and 1.2, respectively. The length of the source access region is $1.2 \mu \mathrm{m}$.

where $V_{\mathrm{SNZ}}$ is the voltage drop across the SNZ zone. The drain access region can be treated similarly but both zones Z4 and Z5 must be considered. Figure 6 shows the nonlinear source resistance as a function of the chancel conductance current with a source access region of $1.2 \mu \mathrm{m}$. The values of beta are $2,1.5$, and 1.2 , respectively.

6.2. Power Performance Simulated by MWO. An AlGaN/GaN HFET power amplifier is simulated using a single-tone power sweep. The amplifier was biased at a drain bias of $V_{\mathrm{ds}}=$ $28 \mathrm{v}$ and operated in class B at a fundamental frequency of $f=2.14 \mathrm{GHz}$. Three harmonics were used in the simulation and the corresponding three pairs of harmonic impedances for both input and output sides are tuned to fit the experimental measurement. In the initial simulation, we did not take into account the reactance components of the device, so for the harmonic impedances tuning, we also excluded the effects of the reactance. Figure 7 shows the simulated large-signal RF performance compared to the measured data, including output power, power gain, and PAE. The inset of Figure 7 shows the results without considering the channel breakdown effects. As shown in the inset, we observe that when input power increases to around $13 \mathrm{dBm}$, there is an increasing discrepancy between the simulated and measurement RF performance, most apparent in PAE, which increases with increasing input power. PAE becomes unacceptably higher than the measured PAE, due to the onset of channel breakdown in the experimental device. Neglecting breakdown effects causes the model to dramatically overestimate the PAE under large RF drive conditions. When the drain-to-source breakdown effect is considered, the results agree very well with the measured data for the input power ranging from $-8 \mathrm{dbM}$ to $20 \mathrm{dBm}$.

As is evident in Figure 7, channel breakdown is crucial for understanding the large-signal RF performance, and particularly the maximum-power-added efficiency of a

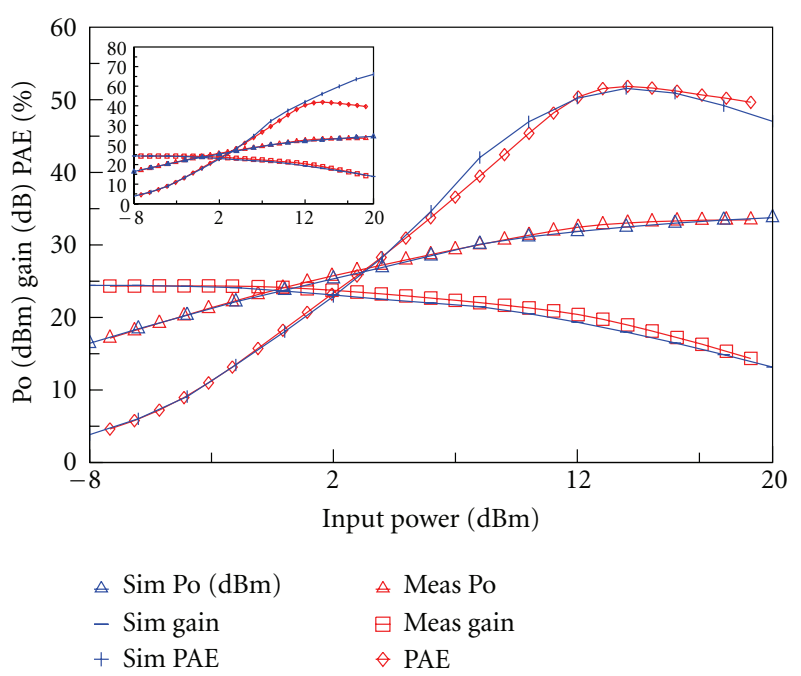

FIGURE 7: Large signal simulation results including output power, power gain and PAE obtained from the HFET model comparison to the measurement data, with and without (insertion graph) channel break down model. ("Red" is measurement data, "blue" is simulation results).

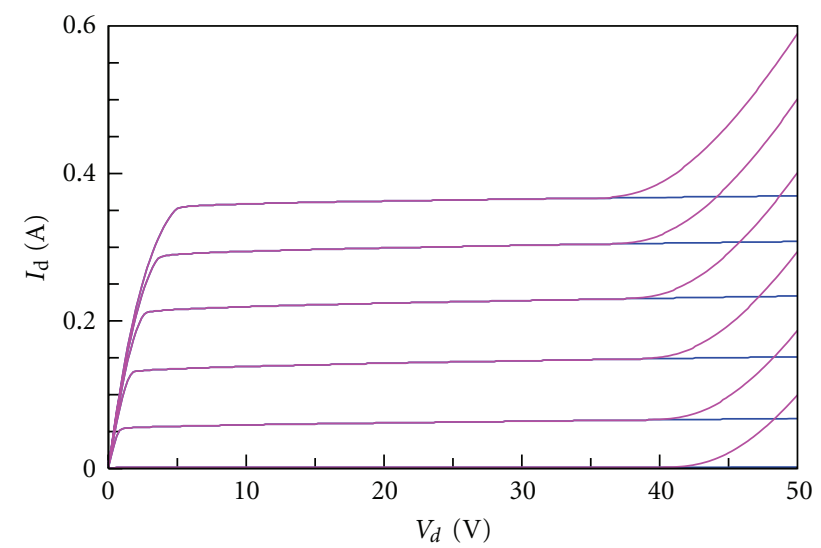

FIGURE 8: Current-voltage characteristics for a $0.8 \mu \mathrm{m}$ HFET with (red lines) and without (blue lines) breakdown computed by MWO.

microwave power amplifier. For sufficiently large terminal voltage, $V_{\mathrm{d} s}$, impact ionization occurs in the conducting channel of the HFET and the avalanche generated current opposes further increase of $V_{\mathrm{ds}}$. If the temperature rise within the device is controlled and limited, channel breakdown is reversible over the RF cycle and the device is not damaged. $\mathrm{RF}$ breakdown is, in fact, a fundamental phenomenon that limits the RF performance of these devices under high power operation. Breakdown in $\mathrm{AlGaN} / \mathrm{GaN}$ transistors occurs in a more gradual manner than in GaAs MESFETs. That is, avalanche ionization occurs in the conducting channel with the generated electrons adding to the channel current, thereby creating a gradual increase in channel current since ionization only occurs during the peak voltage period of the RF cycle when the channel field briefly rises above the breakdown electric field. Since this only occurs during a short 


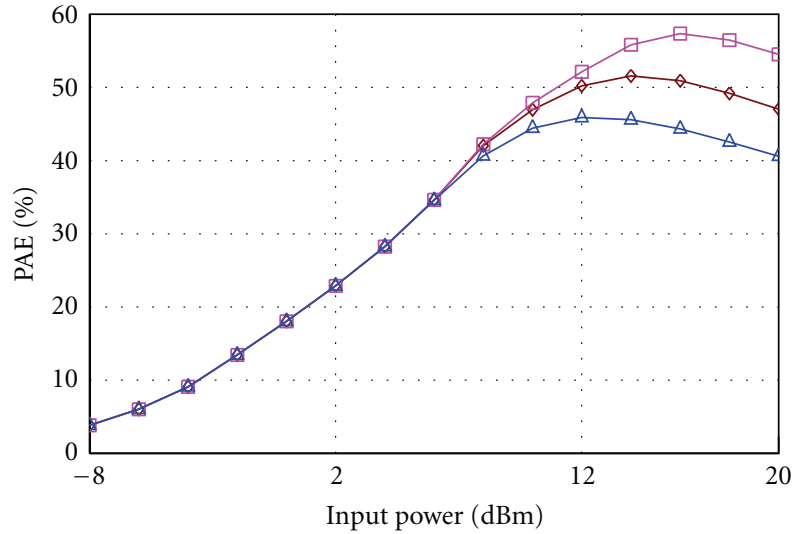

Figure 9: PAE with different breakdown voltage.

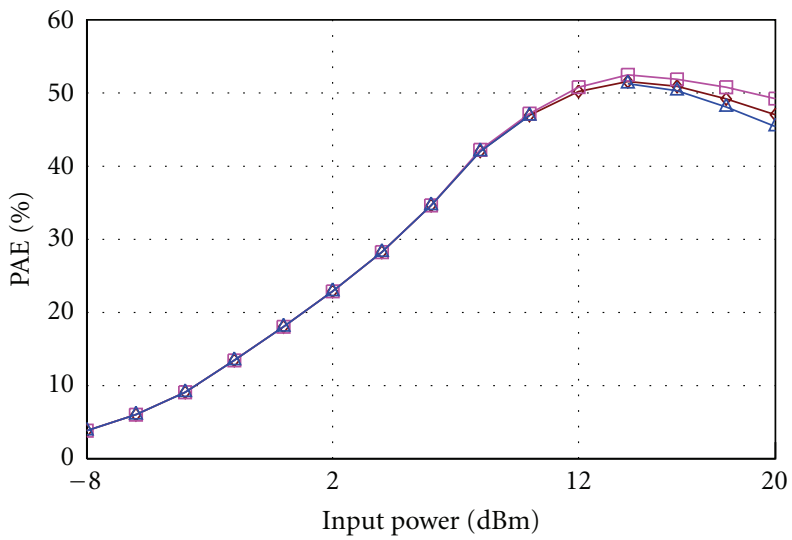

Figure 10: PAE with different breakdown resistance.

period of the RF cycle, a pulse of generated charge occurs, which limits the ionization-generated current increase. Gate breakdown is the dominant RF breakdown mechanism in traditional GaAs MESFETs [27] but does not generally occur in $\mathrm{AlGaN} / \mathrm{GaN}$ HFETs. Experimental data reveals that gate leakage does occur in AlGaN/GaN HFETs [28], but the leakage is caused by electron tunneling from the gate electrode and conduction occurs along the surface area of the HFET between the gate and drain electrodes. Gate leakage affects HFET reliability, introduces transient effects, and is explained elsewhere [29].

6.3. Maximum PAE and Breakdown Resistance. Figure 8 shows the dc current-voltage characteristics with and without breakdown. As indicated, once breakdown occurs, the increase in channel current is gradual with drain voltage.

The effect of the breakdown voltage on large-signal PAE is shown in Figure 9. Increases in the channel breakdown voltage will improve PAE. Figure 10 shows the effect of the breakdown resistance, defined as the slope of the dynamic $I$ - $V$ characteristic once breakdown occurs. For a breakdown voltage of $39 \mathrm{~V}$, when input powers are below $12 \mathrm{dBm}$, the breakdown resistance does not significantly affect the PAE.

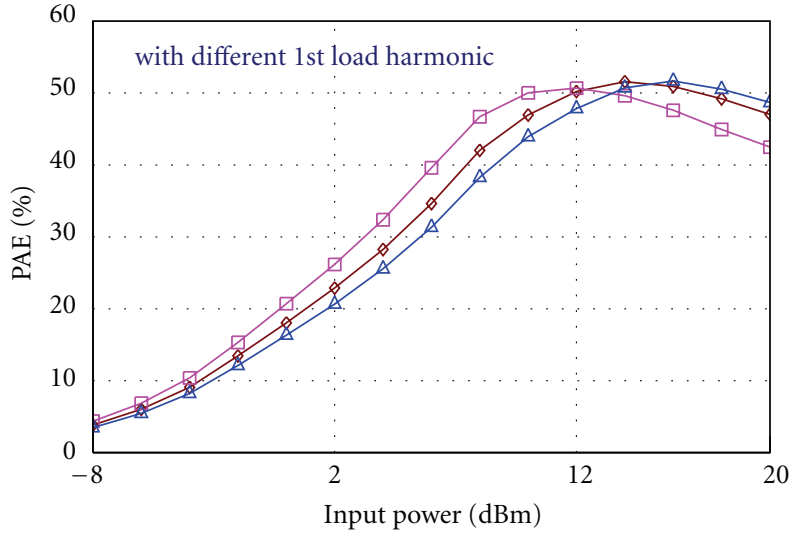

Figure 11: PAE with different 1st load harmonics (real part).

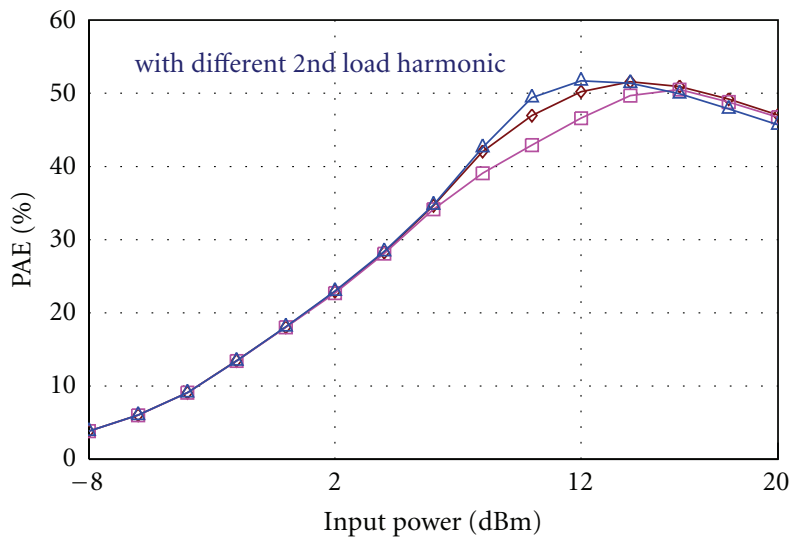

FIgURE 12: PAE with different second load harmonics (real part).

After the input power exceeds $12 \mathrm{dBm}$, the PAE increases with increasing resistance.

6.4. Maximum PAE and Load Resistance at Fundamental and Second Harmonic Frequency. The harmonic impedances of the matching network affect the large signal outputs. The effect of the impedances at the first and second harmonics is shown in Figures 11 and 12.

\section{Conclusion}

A new physics-based compact model for AlGaN/GaN HFETs has been developed. The new model is based upon separating the conducting channel into various zones determined by the physical operation principles that dominate in each zone. A simplified set of the semiconductor device equations are applied to each zone and the zones are then interfaced by forcing electric field and potential continuity at the zone interfaces. In this manner, a complete model for the device can be constructed. The compact model is suitable for integration into readily available harmonic-balance circuit simulators. The model has been formulated in Verilog-A and is integrated into the harmonic-balance simulator Microwave 
Office offered by AWR. However, the new compact model can be employed in any readily available circuit simulator.

The new model permits the $\mathrm{dc}$ and RF performance of the device to be determined as a function of device design parameters such as material parameters, the physical dimensions and doping concentrations of the various layers, and the charge transport characteristics. About twenty parameters are required to define the model, but most of these consist of handbook data. The new model does not require the parameter extraction procedures commonly employed to determine equivalent-circuit-based models. The new model, therefore, requires significantly less effort to define.

The new model has been calibrated and verified by comparison with measured data for experimental AlGaN/GaN HFET amplifiers operating at S-band. Excellent agreement between simulated and measured dc and large-signal RF data is obtained.

\section{Acknowledgment}

This work was supported by ARO grant DAA019-03-1-0148 and by ONR grant N0014-05-0419.

\section{References}

[1] J. C. Zolper, "Wide bandgap semiconductor microwave technologies: from promise to practice," in Proceedings of the IEEE International Devices Meeting (IEDM '99), pp. 389-392, December 1999.

[2] R. J. Trew, "SiC and GaN transistors-Is there one winner for microwave power applications?" Proceedings of the IEEE, vol. 90, no. 6, pp. 1032-1047, 2002.

[3] M. A. Khan, A. Bhattarai, J. N. Kuznia, and D. T. Olson, "High electron mobility transistor based on a GaN-AlxGa (1-x)N heterojunction," Applied Physics Letters, vol. 63, no. 9, pp. 1214-1215, 1993.

[4] Y. F. Wu, B. P. Keller, P. Fini et al., "High Al-content AlGaN/ GaN MODFET's for ultrahigh performance," IEEE Electron Device Letters, vol. 19, no. 2, pp. 50-53, 1998.

[5] W. Lu, J. Yang, M. A. Khan, and I. Adesida, "AlGaN/GaN HEMTs on $\mathrm{SiC}$ with over $100 \mathrm{GHz}$ fT and low microwave noise," IEEE Transactions on Electron Devices, vol. 48, no. 3, pp. 581-585, 2001.

[6] Y. Cordier, J. C. Moreno, N. Baron et al., "Demonstration of AlGaN/GaN high-electron-mobility transistors grown by molecular beam epitaxy on Si(110)," IEEE Electron Device Letters, vol. 29, no. 11, pp. 1187-1189, 2008.

[7] I. Angelov, H. Zirath, and N. Rorsman, "A new empirical nonlinear model for HEMT-devices," in Proceedings of the IEEE MTT-S International Microwave Symposium Digest Part 3, pp. 1583-1586, 1992.

[8] I. Angelov, L. Bengtsson, and M. Garcia, "Extensions of the chalmers nonlinear HEMT and MESFET model," IEEE Transactions on Microwave Theory and Techniques, vol. 44, no. 10, pp. 1664-1674, 1996.

[9] Silvaco Manual, Silvaco Data Systems Inc., Santa Clara, Calif, USA.

[10] A. Asgari, M. Kalafi, and L. Faraone, "A quasi-two-dimensional charge transport model of AlGaN/GaN high electron mobility transistors (HEMTs)," Physica E, vol. 28, no. 4, pp. 491-499, 2005.
[11] H. Ahn and M. El Nokali, "Analytical model for high electron mobility transistors," IEEE Transactions on Electron Devices, vol. 41, no. 6, pp. 874-878, 1994.

[12] R. Singh and C. M. Snowden, "A quasi-two-dimensional HEMT model for DC and microwave simulation," IEEE Transactions on Electron Devices, vol. 45, no. 6, pp. 1165-1169, 1998.

[13] J. D. Albrecht, P. P. Ruden, S. C. Binari, and M. G. Ancona, "AlGaN/GaN heterostructure field-effect transistor model including thermal effects," IEEE Transactions on Electron Devices, vol. 47, no. 11, pp. 2031-2036, 2000.

[14] T. H. Yu and K. F. Brennan, "Theoretical study of a GaNAlGaN high electron mobility transistor including a nonlinear polarization model," IEEE Transactions on Electron Devices, vol. 50, no. 2, pp. 315-323, 2003.

[15] A. Koudymov, M. S. Shur, G. Simin et al., "Analytical HFET $\mathrm{I}-\mathrm{V}$ model in presence of current collapse," IEEE Transactions on Electron Devices, vol. 55, no. 3, pp. 712-720, 2008.

[16] X. Cheng, M. Li, and Y. Wang, "Physics-Based Compact Model for AlGaN/GaN MODFETs With Close-Formed I- V and C- V Characteristics," IEEE Transactions on Electron Devices, vol. 36, no. 2, pp. 231-239, 1988.

[17] M. W. Shin, R. J. Trew, and G. L. Bilbro, "High temperature dc and RF performance of p-type diamond MESFET: comparison with N-type GaAs MESFET," IEEE Electron Device Letters, vol. 15, no. 8, pp. 292-294, 1994.

[18] C. W. Hatfield, G. L. Bilbro, S. T. Allen, and J. W. Palmour, "DC I-V characteristics and RF performance of a $4 \mathrm{H}-\mathrm{SiC}$ JFET at 773 K," IEEE Transactions on Electron Devices, vol. 45, no. 9 , pp. 2072-2074, 1998.

[19] M. A. Khatibzadeh and R. J. Trew, "LARGE-SIGNAL, ANALYTIC MODEL FOR THE GAAS MESFET,” IEEE Transactions on Microwave Theory and Techniques, vol. 36, no. 2, pp. 231238, 1988.

[20] R. J. Trew, Y. Liu, G. L. Bilbro, W. Kuang, R. Vetury, and J. B. Shealy, "Nonlinear source resistance in high-voltage microwave alGaN/gaN HFETs," IEEE Transactions on Microwave Theory and Techniques, vol. 54, no. 5, pp. 2061-2067, 2006.

[21] H. Yin, D. Hou, G. L. Bilbro, and R. J. Trew, "Harmonie balance simulation of a new physics based model of the AlGaN/GaN HFET," in Proceedings of the IEEE International Microwave Symposium Digest, (MTT-S '08), pp. 1425-1428, 2008.

[22] C. Canali, G. Majni, R. Minder, and G. Ottaviani, "Electron and hole drift velocity measurements in silicon and their empirical relation to electric field and temperature," IEEE Transactions on Electron Devices, vol. 22, no. 11, pp. 10451047, 1975.

[23] M. Farahmand, C. Garetto, E. Bellotti et al., "Monte Carlo simulation of electron transport in the III-nitride Wurtzite phase materials system: binaries and ternaries," IEEE Transactions on Electron Devices, vol. 48, no. 3, pp. 535-542, 2001.

[24] V. M. Polyakov and F. Schwierz, "Influence of electron mobility modeling on dc I-V characteristics of WZ-GaN MESFET," IEEE Transactions on Electron Devices, vol. 48, no. 3, pp. 512$516,2001$.

[25] B. K. Ridley, "Coupled surface and channel transport in semiconductor heterostructures," Journal of Applied Physics, vol. 90, no. 12, pp. 6135-6139, 2001.

[26] O. Ambacher, J. Smart, J. R. Shealy et al., "Two-dimensional electron gases induced by spontaneous and piezoelectric polarization charges in N- And Ga-face AIGaN/GaN heterostructures," Journal of Applied Physics, vol. 85, no. 6, pp. 3222-3233, 1999. 
[27] T. A. Winslow and R. J. Trew, "Principles of large-signal MESFET operation," IEEE Transactions on Microwave Theory and Techniques, vol. 42, no. 6, pp. 935-942, 1994.

[28] R. J. Trew, Y. Liu, W. Kuang et al., "RF breakdown and largesignal modeling of AlGaN/GaN HFET's," in Proceedings of the IEEE International Microwave Symposium Digest (MTT-S '06), pp. 643-646, San Francisco, Calif, USA, 2006.

[29] R. J. Trew, D. S. Green, and J. B. Shealy, "AlGaN/GaN HFET reliability," IEEE Microwave Magazine, vol. 10, pp. 116-127, 2009. 

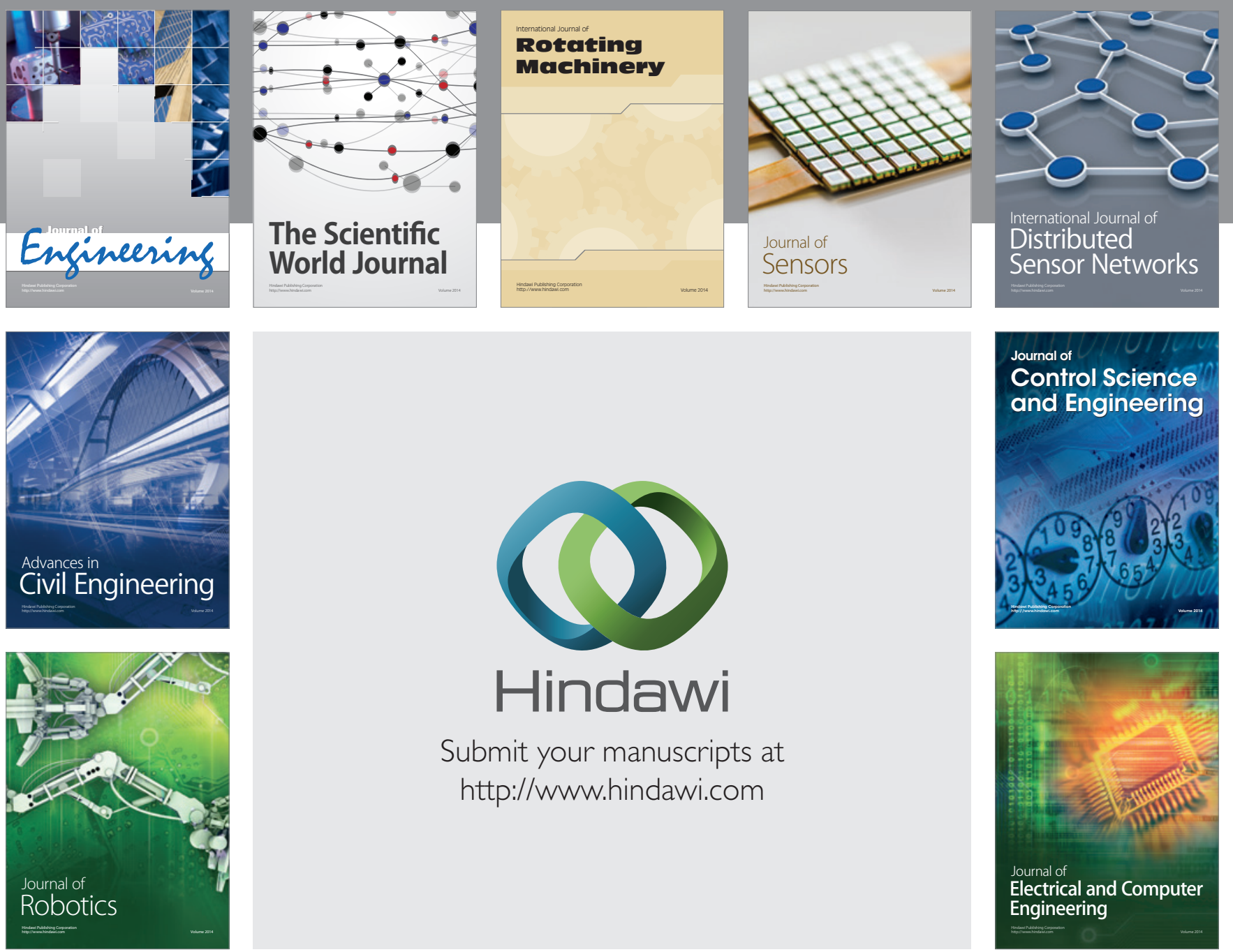

Submit your manuscripts at

http://www.hindawi.com
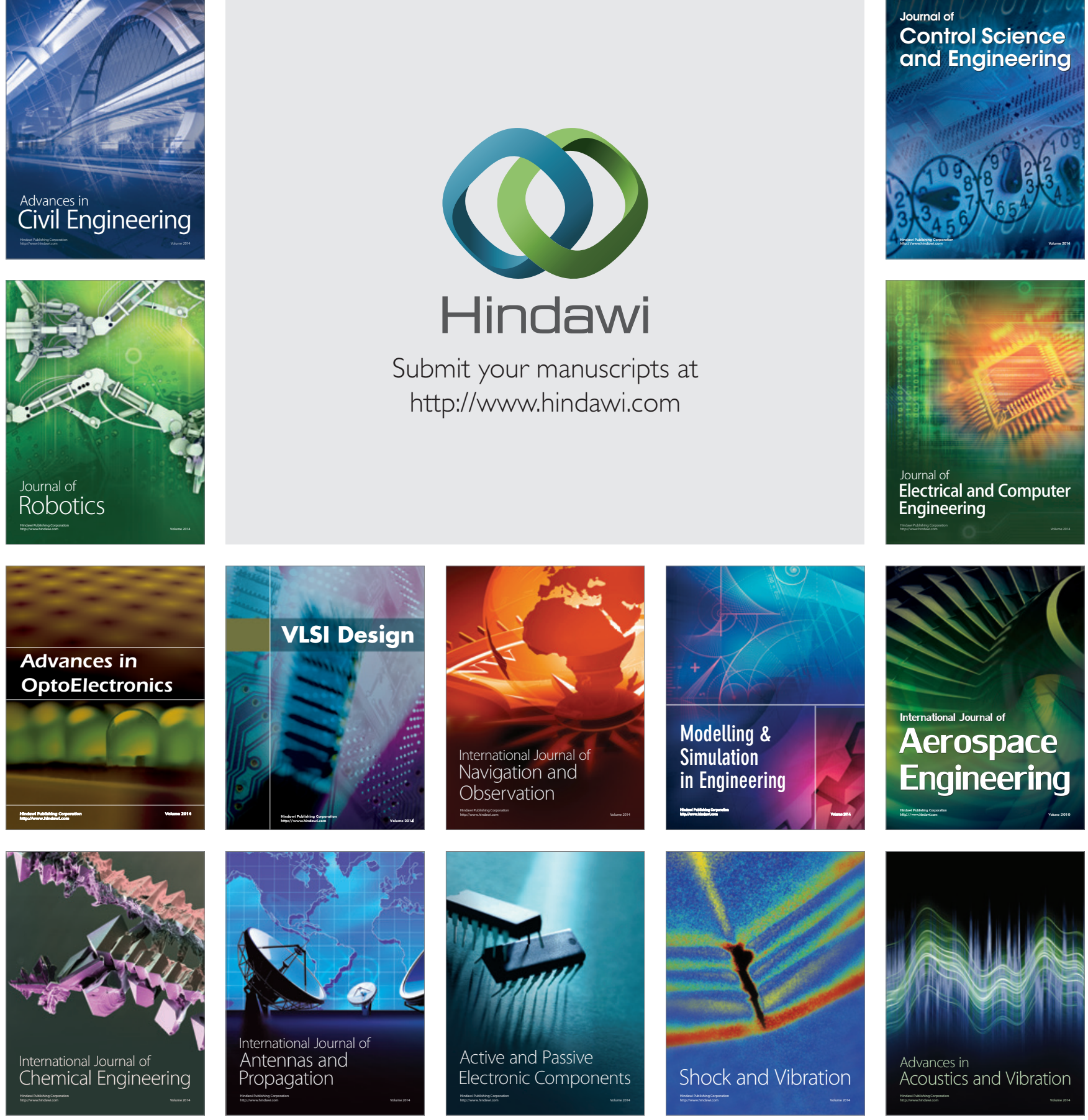